\title{
Thompson's Group and Public Key Cryptography
}

\author{
Vladimir Shpilrain ${ }^{1, \star}$ and Alexander Ushakov ${ }^{2}$ \\ 1 Department of Mathematics, The City College of New York, New York, NY 10031 \\ shpilrain@yahoo.com \\ 2 Department of Mathematics, CUNY Graduate Center, New York, NY 10016 \\ aushakov@mail.ru
}

\begin{abstract}
Recently, several public key exchange protocols based on symbolic computation in non-commutative (semi)groups were proposed as a more efficient alternative to well established protocols based on numeric computation. Notably, the protocols due to Anshel-Anshel-Goldfeld and Ko-Lee et al. exploited the conjugacy search problem in groups, which is a ramification of the discrete logarithm problem. However, it is a prevalent opinion now that the conjugacy search problem alone is unlikely to provide sufficient level of security no matter what particular group is chosen as a platform.

In this paper we employ another problem (we call it the decomposition problem), which is more general than the conjugacy search problem, and we suggest to use R. Thompson's group as a platform. This group is well known in many areas of mathematics, including algebra, geometry, and analysis. It also has several properties that make it fit for cryptographic purposes. In particular, we show here that the word problem in Thompson's group is solvable in almost linear time.
\end{abstract}

\section{Introduction}

One of the possible generalizations of the discrete logarithm problem to arbitrary groups is the so-called conjugacy search problem: given two elements $a, b$ of a group $G$ and the information that $a^{x}=b$ for some $x \in G$, find at least one particular element $x$ like that. Here $a^{x}$ stands for $x^{-1} a x$. The (alleged) computational difficulty of this problem in some particular groups (namely, in braid groups) has been used in several group based cryptosystems, most notably in [1] and [6]. It seems however now that the conjugacy search problem alone is unlikely to provide sufficient level of security; see [7] and [8] for explanations.

In this paper we employ another problem, which generalizes the conjugacy search problem, but at the same time resembles the factorization problem which is at the heart of the RSA cryptosystem. This problem which some authors (see e.g. [3], [6]) call the decomposition problem is as follows:

Given an element $w$ of a (semi)group $G$, a subset $A \subseteq G$ and an element $x \cdot w \cdot y$, find elements $x^{\prime}, y^{\prime} \in A$ such that $x^{\prime} \cdot w \cdot y^{\prime}=x \cdot w \cdot y$.

\footnotetext{
* Research of the first author was partially supported by the NSF grant DMS-0405105.

J. Ioannidis, A. Keromytis, and M.Yung (Eds.): ACNS 2005, LNCS 3531, pp. 151-163, 2005.

(C) Springer-Verlag Berlin Heidelberg 2005
} 
The conjugacy search problem (more precisely, its subgroup-restricted version used in [6]) is a special case of the decomposition problem if one takes $x=y^{-1}$.

The usual factorization problem for integers used in the RSA cryptosystem is also a special case of the decomposition problem if one takes $w=1$ and $G=\mathbf{Z}_{p}^{*}$, the multiplicative (semi)group of integers modulo $p$. It is therefore conceivable that with more complex (semi)groups used as platforms, the corresponding cryptosystem may be more secure. At the same time, in the group that we use in this paper (R. Thompson's group), computing (the normal form of) a product of elements is faster than in $\mathbf{Z}_{p}^{*}$.

A key exchange protocol based on the general decomposition problem is quite straightforward (see e.g. [6]): given two subsets $A, B \subseteq G$ such that $a b=b a$ for any $a \in A, b \in B$, and given a public element $w \in G$, Alice selects private $a_{1}, a_{2} \in A$ and sends the element $a_{1} w a_{2}$ to Bob. Similarly, Bob selects private $b_{1}, b_{2} \in B$ and sends the element $b_{1} w b_{2}$ to Alice. Then Alice computes $K_{A}=$ $a_{1} b_{1} w b_{2} a_{2}$, and Bob computes $K_{B}=b_{1} a_{1} w a_{2} b_{2}$. Since $a_{i} b_{i}=b_{i} a_{i}$ in $G$, one has $K_{A}=K_{B}=K$ (as an element of $G$ ), which is now Alice's and Bob's common secret key.

In this paper, we suggest the following modification of this protocol which appears to be more secure (at least for our particular choice of the platform) against so-called "length based" attacks (see e.g. [4], [5]), according to our experiments (see our Section 3). Given two subsets $A, B \subseteq G$ such that $a b=b a$ for any $a \in A, b \in B$, and given a public element $w \in G$, Alice selects private $a_{1} \in A$ and $b_{1} \in B$ and sends the element $a_{1} w b_{1}$ to Bob. Bob selects private $b_{2} \in B$ and $a_{2} \in A$ and sends the element $b_{2} w a_{2}$ to Alice. Then Alice computes $K_{A}=a_{1} b_{2} w a_{2} b_{1}$, and Bob computes $K_{B}=b_{2} a_{1} w b_{1} a_{2}$. Since $a_{i} b_{i}=b_{i} a_{i}$ in $G$, one has $K_{A}=K_{B}=K$ (as an element of $G$ ), which is now Alice's and Bob's common secret key.

The group that we suggest to use as the platform for this protocol is Thompson's group $F$ well known in many areas of mathematics, including algebra, geometry, and analysis. This group is infinite non-abelian. For us, it is important that Thompson's group has the following nice presentation in terms of generators and defining relations:

$$
F=\left\langle x_{0}, x_{1}, x_{2}, \ldots \mid x_{i}^{-1} x_{k} x_{i}=x_{k+1}(k>i)\right\rangle .
$$

This presentation is infinite. There are also finite presentations of this group; for example,

$$
F=\left\langle x_{0}, x_{1}, x_{2}, x_{3}, x_{4} \mid x_{i}^{-1} x_{k} x_{i}=x_{k+1}(k>i, k<4)\right\rangle,
$$

but it is the infinite presentation above that allows for a convenient normal form, so we are going to use that presentation in our paper.

For a survey on various properties of Thompson's group, we refer to [2]. Here we only give a description of the "classical" normal form for elements of $F$.

The classical normal form for an element of Thompson's group is a word of the form

$$
x_{i_{1}} \ldots x_{i_{s}} x_{j_{t}}^{-1} \ldots x_{j_{1}}^{-1}
$$

such that the following two conditions are satisfied: 
(NF1) $i_{1} \leq \ldots \leq i_{s}$ and $j_{1} \leq \ldots \leq j_{t}$

(NF2) if both $x_{i}$ and $x_{i}^{-1}$ occur, then either $x_{i+1}$ or $x_{i+1}^{-1}$ occurs, too.

We say that a word $w$ is in seminormal form if it is of the form (2) and satisfies (NF1).

We show in Section 4 that the time complexity of reducing a word of length $n$ to the normal form in Thompson's group is $O(|n| \log |n|)$, i.e., is almost linear in $n$.

Another advantage of cryptographic protocols based on symbolic computation over those based on computation with numbers is the possibility to generate a random word one symbol at a time. For example, in RSA, one uses random prime numbers which obviously cannot be generated one digit at a time but rather have to be precomputed, which limits the key space unless one wants to sacrifice the efficiency. We discuss key generation in more detail in our Section 3.

Acknowledgments. We are grateful to V. Guba for helpful comments and to R. Haralick for making a computer cluster in his lab available for our computer experiments.

\section{The Protocol}

Let $F$ be Thompson's group given by its standard infinite presentation (1) and $s \in \mathbb{N}$ a positive integer. Define sets $A_{s}$ and $B_{s}$ as follows. The set $A_{s}$ consists of elements whose normal form is of the type

$$
x_{i_{1}} \ldots x_{i_{m}} x_{j_{m}}^{-1} \ldots x_{j_{1}}^{-1},
$$

i.e. positive and negative parts are of the same length $m$, and

$$
i_{k}-k<s \text { and } j_{k}-k<s \text { for every } k=1, \ldots, s .
$$

The set $B_{s}$ consists of elements represented by words in generators $x_{s+1}, x_{s+2}, \ldots$.. Obviously, $B_{s}$ is a subgroup of $F$.

Proposition 1. Let $a \in A_{s}$ and $b \in B_{s}$. Then $a b=b a$ in the group $F$.

Proof. Let $a=x_{i_{1}} \ldots x_{i_{m}} x_{j_{m}}^{-1} \ldots x_{j_{1}}^{-1}$ and $b=x_{k_{1}}^{\varepsilon_{1}} \ldots x_{k_{l}}^{\varepsilon_{l}}$ where $k_{q}>s$ for every $q=1, \ldots, l$. By induction on $l$ and $m$ it is easy to show that in the group $F$ one has

$$
a b=b a=x_{i_{1}} \ldots x_{i_{m}} \delta_{m}(b) x_{j_{m}}^{-1} \ldots x_{j_{1}}^{-1},
$$

where $\delta_{M}$ is the operator that increases indices of all generators by $M$ (see also our Section 4). This establishes the claim.

Proposition 2. Let $s \geq 2$ be an integer. The set $A_{s}$ is a subgroup of $F$ generated by $x_{0} x_{1}^{-1}, \ldots, x_{0} x_{s}^{-1}$. 
Proof. The set $A_{s}$ contains the identity and is clearly closed under taking inversions, i.e., $A_{s}=A_{s}^{-1}$. To show that $A_{s}$ is closed under multiplication we take two arbitrary normal forms from $A_{s}$ :

$$
u=x_{i_{1}} \ldots x_{i_{m}} x_{j_{m}}^{-1} \ldots x_{j_{1}}^{-1}
$$

and

$$
v=x_{p_{1}} \ldots x_{p_{l}} x_{q_{l}}^{-1} \ldots x_{q_{1}}^{-1}
$$

and show that the normal form of $u v$ belongs to $A_{s}$. First, note that since the numbers of positive and negative letters in $u v$ are equal, the lengths of the positive and negative parts in the normal form of $u v$ will be equal, too (see the rewriting system in the beginning of our Section 4). Thus, it remains to show that the property (3) of indices in the normal form of $u v$ is satisfied. Below we sketch the proof of this claim.

Consider the subword in the middle of the product $u v$ marked below:

$$
u v=x_{i_{1}} \ldots x_{i_{m}}\left(x_{j_{m}}^{-1} \ldots x_{j_{1}}^{-1} x_{p_{1}} \ldots x_{p_{l}}\right) x_{q_{l}}^{-1} \ldots x_{q_{1}}^{-1}
$$

and find a seminormal form for it using relations of $F$ (move positive letters to the left and negative letters to the right starting in the middle of the subword). We refer the reader to Algorithm 2 in Section 4 for more information on how this can be done. Denote the obtained word by $w$. The word $w$ is the product of a positive and a negative word: $w=p n$. By induction on $l+m$ one can show that both $p$ and $n$ satisfy the condition (3).

Then we find normal forms for words $p$ and $n$ using relations of $F$ (for $p$ move letters with smaller indices to the left of letters with bigger indices, and for $n$ move letters with smaller indices to the right of letters with bigger indices). By induction on the number of operations thus performed, one can show that the obtained words $p^{\prime}$ and $n^{\prime}$ satisfy the condition (3). Therefore, the word $w^{\prime}=p^{\prime} n^{\prime}$ is a seminormal form of $u v$ satisfying the condition (3).

Finally, we remove those pairs of generators in $w^{\prime}$ that contradict the property (NF2) (we refer the reader to our Algorithm 5 for more information). Again, by induction on the number of "bad pairs", one can show that the result will satisfy the condition (3). Therefore, $u v$ belongs to $A_{s}$, i.e., $A_{s}$ is closed under multiplication, and therefore, $A_{s}$ is a subgroup.

Now we show that the set of words $\left\{x_{0} x_{1}^{-1}, \ldots, x_{0} x_{s}^{-1}\right\}$ generates the subgroup $A_{s}$. Elements $\left\{x_{0} x_{1}^{-1}, \ldots, x_{0} x_{s}^{-1}\right\}$ clearly belong to $A_{s}$. To show the inclusion $A_{s} \leq\left\langle x_{0} x_{1}^{-1}, \ldots, x_{0} x_{s}^{-1}\right\rangle$, we construct the Schreier graph of $\left\langle x_{0} x_{1}^{-1}, \ldots\right.$, $\left.x_{0} x_{s}^{-1}\right\rangle$ (depicted in Figure 1) and see that any word from $A_{s}$ belongs to the subgroup on the right.

Now we give a formal description of the protocol based on the decomposition problem mentioned in the Introduction.

(0) Fix two positive integers $s, M$ and a word $w=w\left(x_{0}, x_{1}, \ldots\right)$.

(1) Alice randomly selects private elements $a_{1} \in A_{s}$ and $b_{1} \in B_{s}$. Then she reduces the element $a_{1} w b_{1}$ to the normal form and sends the result to Bob. 


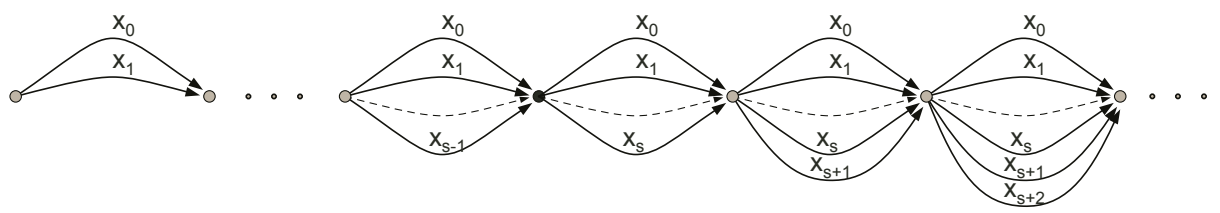

Fig. 1. The Schreier graph of the subgroup $H=\left\langle x_{0} x_{1}^{-1}, \ldots, x_{0} x_{s}^{-1}\right\rangle$. The black dot denotes the right coset corresponding to $H$.

(2) Bob randomly selects private elements $b_{2} \in B_{s}$ and $a_{2} \in A_{s}$. Then he reduces the element $b_{2} w a_{2}$ to the normal form and sends the result to Alice.

(3) Alice computes $K_{A}=a_{1} b_{2} w a_{2} b_{1}=b_{2} a_{1} w b_{1} a_{2}$, and Bob computes $K_{B}=$ $b_{2} a_{1} w b_{1} a_{2}$. Since $a_{i} b_{i}=b_{i} a_{i}$ in $F$, one has $K_{A}=K_{B}=K$ (as an element of $F$ ), which is now Alice's and Bob's common secret key.

\section{Parameters and Key Generation}

In practical key exchange we suggest to choose the following parameters.

(1) Select (randomly and uniformly) the parameter $s$ from the interval $[3,8]$ and the parameter $M$ from the set $\{256,258, \ldots, 318,320\}$.

(2) Select the "base" word $w$ as a product of generators

$$
S_{W}=\left\{x_{0}, x_{1}, \ldots, x_{s+2}\right\}
$$

and their inverses. This is done the following way. We start with the empty word $v_{0}$. When we have a current word $v_{i}$, we multiply it on the right by a generator from $S_{B}^{ \pm 1}$ and compute the normal form of the product. The obtained word is denoted by $v_{i+1}$. We continue this process until the obtained word $v_{i+1}$ has length $M$.

(3) Select $a_{1}$ and $a_{2}$ as products of words from

$$
S_{A}=\left\{x_{0} x_{1}^{-1}, \ldots, x_{0} x_{s}^{-1}\right\}
$$

and their inverses. This is done essentially the same way as above for $w$. We start with the empty word $u_{0}$. Let $u_{i}$ be the currently constructed word of length less than $M$. We multiply $u_{i}$ on the right by a randomly chosen word from $S_{A}^{ \pm 1}$ and compute the normal form of the product. Denote the obtained normal form by $u_{i+1}$. Continue this process until the obtained word $u_{i+1}$ has length $M$.

(4) Select $b_{1}$ and $b_{2}$ as products of generators from

$$
S_{B}=\left\{x_{s+1}, x_{s+2}, \ldots, x_{2 s}\right\}
$$

and their inverses. To do that, start with the empty word $v_{0}$. Multiply a current word $v_{i}$ on the right by a generator from $S_{B}^{ \pm 1}$ and compute the normal form of 
the product. Denote the obtained word by $v_{i+1}$. Continue this process until the obtained word $v_{i+1}$ has length $M$.

We would like to point out that the key space in the proposed scheme is exponential in $M$; it is easy to see that $\left|A_{s}(M)\right| \geq \sqrt{2}^{M}$.

The parameters above were chosen in such a way to prevent a length-based attack. Note that for Thompson's group, a length-based attack could be a threat since the normal form of any element represents a geodesic in the Cayley graph of $F$. Since ideas behind length-based attacks were never fully described, we present below a typical algorithm (adapted to our situation) implementing such an attack (Algorithm 1).

Define a directed labelled graph $\Gamma=(V(\Gamma), E(\Gamma))$ as follows:

- The set of vertices $V(\Gamma)$ corresponds to the set of all elements of the group F.

- The set of edges $E(\Gamma)$ contains edges $v_{1} \stackrel{\left(w_{1}, w_{2}\right)}{\longrightarrow} v_{2}$ such that $v_{2}=w_{1} v_{1} w_{2}$ in the group $F$, with labels of two types:

- $\left(w_{1}, 1\right)$, where $w_{1} \in S_{A}^{ \pm 1}$.

- $\left(1, w_{2}\right)$, where $w_{2} \in S_{B}^{A 1}$.

For an element $w \in F$ denote by $\Gamma_{w}$ the connected component of $\Gamma$ containing $w$. From the description of the protocol it follows that $w$ and the element $w^{\prime}=a_{1} w b_{1}$ transmitted by Alice to Bob belong to $\Gamma_{w}=\Gamma_{w^{\prime}}$, and breaking Alice's key is equivalent to finding a label of a path from $w$ to $w^{\prime}$ in $\Gamma_{w}$.

To test our protocol, we performed a series of experiments. We randomly generated keys (as described above) and ran Algorithm 1 (see below) on them. Algorithm 1 keeps constructing $\Gamma_{w}$ and $\Gamma_{w^{\prime}}$ until a shared element is found. The sets $S_{w}$ and $S_{w^{\prime}}$ in the algorithm accumulate constructed parts of the graphs $\Gamma_{w}$ and $\Gamma_{w^{\prime}}$. The sets $M_{w} \subset S_{w}$ and $M_{w^{\prime}} \subseteq S_{w^{\prime}}$ are called the sets of marked vertices and are used to specify vertices that are worked out.

Algorithm 1 (Length-based attack)

InPUT. The original public word $w$ and the word $w^{\prime}$ transmitted by Alice.

Output. A pair of words $x_{1} \in S_{A}, x_{2} \in S_{B}$ such that $w^{\prime}=x_{1} w x_{2}$.

Initialization. Put $S_{w}=\{w\}, S_{w^{\prime}}=\left\{w^{\prime}\right\}, M_{w}=\emptyset, M_{w^{\prime}}=\emptyset$.

Computations.

A. Find a shortest word $u \in S_{w} \backslash M_{w}$.

B. Multiply $u$ by elements $S_{A}^{ \pm 1}$ on the left and by elements $S_{B}^{ \pm 1}$ on the right and add each result into $S_{w}$ with the edges labelled accordingly.

C. Add u into $M_{w}$.

D. Perform the steps $A-C$ with $S_{w}$ and $M_{w}$ replaced by $S_{w^{\prime}}$ and $M_{w^{\prime}}$, respectively.

E. If $S_{w} \cap S_{w^{\prime}}=\emptyset$ then goto $A$.

F. If there is $\bar{w} \in S_{w} \cap S_{w^{\prime}}$ then find a path in $S_{w}$ from $w$ to $\bar{w}$ and a path in $S_{w^{\prime}}$ from $\bar{w}$ to $w^{\prime}$. Concatenate them and output the label of the result.

We performed a series of tests implementing this length-based attack; in each test we let the program to run overnight. None of the programs gave a result, i.e., the success rate of the length-based attack in our tests was 0 . 


\section{The Word Problem in Thompson's Group}

In this section, we show that the time complexity of reducing a word of length $n$ to the normal form in Thompson's group $F$ is $O(|n| \log |n|)$, i.e., is almost linear in $n$. Our algorithm is in two independent parts: first we reduce a given word to a seminormal form (Algorithm 4), and then further reduce it to the normal form by eliminating "bad pairs" (Algorithm 5). We also note that crucial for Algorithm 4 is Algorithm 3 which computes a seminormal form of a product of two seminormal forms. Our strategy for computing a seminormal form of a given $w \in F$ is therefore recursive ("divide and conquer"): we split the word $w$ into two halves: $w=w_{1} w_{2}$, then compute seminormal forms of $w_{1}$ and $w_{2}$, and then use Algorithm 3 to compute a seminormal form of $w$.

Recall that Thompson's group $F$ has the following infinite presentation:

$$
F=\left\langle x_{0}, x_{1}, x_{2}, \ldots \mid x_{i}^{-1} x_{k} x_{i}=x_{k+1}(k>i)\right\rangle .
$$

The classical normal form for an element of Thompson's group (see [2] for more information) is described in the Introduction.

Let us denote by $\rho(w)$ the normal form for $w \in F$; it is unique for a given element of $F$. Recall that we say that a word $w$ is in seminormal form if it is of the form (2) and satisfies (NF1) (see the Introduction). A seminormal form is not unique. As usual, for a word $w$ in the alphabet $X$ by $\bar{w}$ we denote the corresponding freely reduced word.

As mentioned above, the normal form for an element of Thompson's group can be computed in two steps:

1) Computation of a seminormal form.

2) Removing "bad pairs", i.e., pairs $\left(x_{i}, x_{i}^{-1}\right)$ for which the property (NF2) fails.

The first part is achieved (Lemma 1) by using the following rewriting system (for all pairs $(i, k)$ such that $i<k)$ :

$$
\begin{array}{ll}
x_{k} x_{i} & \rightarrow x_{i} x_{k+1} \\
x_{k}^{-1} x_{i} & \rightarrow x_{i} x_{k+1}^{-1} \\
x_{i}^{-1} x_{k} & \rightarrow x_{k+1} x_{i}^{-1} \\
x_{i}^{-1} x_{k}^{-1} & \rightarrow x_{k+1}^{-1} x_{i}^{-1}
\end{array}
$$

and, additionally, for all $i \in \mathbb{N}$

$$
x_{i}^{-1} x_{i} \rightarrow 1
$$

We denote this system of rules by $\mathcal{R}$. It is straightforward to check (using the confluence test, see [10, Proposition 3.1]) that $\mathcal{R}$ is confluent. The following lemma is obvious.

Lemma 1. $\mathcal{R}$ terminates with a seminormal form. Moreover, a word is in a seminormal form if and only if it is $\mathcal{R}$-reduced. 
Let us now examine the action of $\mathcal{R}$ more closely. This action is similar to sorting a list of numbers, but with two differences: indices of generators may increase, and some generators may disappear.

By Lemma 1, for any word $w$ in generators of $F$, the final result of rewrites by $\mathcal{R}$ is a seminormal form. Therefore, to compute a seminormal form we implement rewrites by $\mathcal{R}$. We do it in a special manner in Algorithm 3 in order to provide the best performance. For convenience we introduce a parametric function $\delta_{\varepsilon}, \varepsilon \in \mathbb{Z}$, defined on the set of all words in the alphabet $\left\{x_{0}^{ \pm 1}, x_{1}^{ \pm 1}, \ldots\right\}$ by

$$
x_{i}^{ \pm 1} \stackrel{\delta_{\varepsilon}}{\mapsto} x_{i+\varepsilon}^{ \pm 1} .
$$

The function $\delta_{\varepsilon}$ may not be defined for some negative $\varepsilon$ on a given word $w=w\left(x_{i_{1}}^{ \pm 1}, x_{i_{2}}^{ \pm 1}, \ldots\right)$, but when it is used, it is assumed that the function is defined.

\subsection{Merging Seminormal Forms}

Consider first the case where a word $w$ is a product of $w_{1}$ and $w_{2}$ given in seminormal forms. Let $w_{1}=p_{1} n_{1}$ and $w_{2}=p_{2} n_{2}$, where $p_{i}$ and $n_{i}(i=1,2)$ are the positive and negative parts of $w_{i}$. Clearly, one can arrange the rewriting process for $p_{1} n_{1} p_{2} n_{2}$ by $\mathcal{R}$ the following way:

1) Rewrite the subword $n_{1} p_{2}$ of $w$ to a seminormal form $p_{2}^{\prime} n_{1}^{\prime}$. Denote by $w^{\prime}=$ $p_{1} p_{2}^{\prime} n_{1}^{\prime} n_{2}$ the obtained result.

2) Rewrite the positive subword $p_{1} p_{2}^{\prime}$ of $w^{\prime}$ to a seminormal form $p$. Denote by $w^{\prime \prime}=p n_{1}^{\prime} n_{2}$ the obtained result.

3) Rewrite the negative subword $n_{1}^{\prime} n_{2}$ of $w^{\prime \prime}$ to a seminormal form $n$. Denote by $w^{\prime \prime \prime}=p n$ the obtained result.

The word $w^{\prime \prime \prime}=p n$ is clearly in a seminormal form and $w={ }_{F} w^{\prime \prime \prime}$. This process can be depicted as follows:

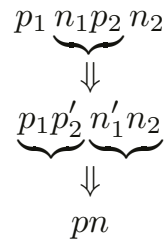

The next algorithm performs the first rewriting step from the scheme above, and the following Lemma 2 asserts that it correctly performs the first step in linear time.

Algorithm 2 (Seminormal form of a product of negative and positive seminormal forms)

Signature. $w=$ Merge $_{-,+}\left(n, p, \varepsilon_{1}, \varepsilon_{2}\right)$.

INPUT. Seminormal forms $n$ and $p$ (where $n=x_{j_{t}}^{-1} \ldots x_{j_{1}}^{-1}$ and $p=x_{i_{1}} \ldots x_{i_{s}}$ ), and numbers $\varepsilon_{1}, \varepsilon_{2} \in \mathbb{Z}$.

OutPut. Seminormal form $w$ such that $w={ }_{F} \delta_{\varepsilon_{1}}(n) \delta_{\varepsilon_{2}}(p)$. 


\section{Computations.}

A) If $s=0$ or $t=0$ then output a product $n p$.

B) If $j_{1}+\varepsilon_{1}=i_{1}+\varepsilon_{2}$ then

1) Compute $w=$ Merge $_{-,+}\left(x_{j_{t}}^{-1} \ldots x_{j_{2}}^{-1}, x_{i_{2}} \ldots x_{i_{s}}, \varepsilon_{1}, \varepsilon_{2}\right)$.

2) Output $w$.

C) If $j_{1}+\varepsilon_{1}<i_{1}+\varepsilon_{2}$ then

1) Compute $w=$ Merge $_{-,+}\left(x_{j_{t}}^{-1} \ldots x_{j_{2}}^{-1}, x_{i_{1}} \ldots x_{i_{s}}, \varepsilon_{1}, \varepsilon_{2}+1\right)$.

2) Output $w x_{j_{1}+\varepsilon_{1}}^{-1}$.

D) If $j_{1}+\varepsilon_{1}>i_{1}+\varepsilon_{2}$ then

1) Compute $w=$ Merge $_{-,+}\left(x_{j_{t}}^{-1} \ldots x_{j_{1}}^{-1}, x_{i_{2}} \ldots x_{i_{s}}, \varepsilon_{1}+1, \varepsilon_{2}\right)$.

2) Output $x_{i_{1}+\varepsilon_{2}} w$.

Lemma 2. For any seminormal forms $n$ and $p$ (where $n=x_{j_{t}}^{-1} \ldots x_{j_{1}}^{-1}$ and $\left.p=x_{i_{1}} \ldots x_{i_{s}}\right)$ and numbers $\varepsilon_{1}, \varepsilon_{2} \in \mathbb{Z}$ the output $w=$ Merge $_{-,+}\left(n, p,, \varepsilon_{1}, \varepsilon_{2}\right)$ of Algorithm 2 is a seminormal form for $\delta_{\varepsilon_{1}}(n) \delta_{\varepsilon_{2}}(p)$. Furthermore, the time complexity required to compute $w$ is bounded by $C(|n|+|p|)$ for some constant $C$.

Proof. Since in each iteration we perform the constant number of elementary steps and in each subsequent iteration the sum $|n|+|p|$ is decreased by one, the time complexity of Algorithm 2 is linear.

We prove correctness of Algorithm 2 by induction on $|n|+|p|$. Assume that $|n|+|p|=0$. Then at step A) we get output $w=n p$ which is an empty word. Clearly, such $w$ is a seminormal form for $n p$, so the base of induction is done.

Assume that $|n|+|p|=N+1$ and for any shorter word the statement is true. Consider four cases. If $|n|=0$ or $|p|=0$ then one of the words is trivial and, obviously, the product $n p$ is a correct output for this case. If $j_{1}+\varepsilon_{1}=i_{1}+\varepsilon_{2}$ then $x_{j_{1}+\varepsilon_{1}}^{-1} x_{i_{1}+\varepsilon_{2}}$ cancels out inside of the product $\delta_{\varepsilon_{1}}(n) \delta_{\varepsilon_{2}}(p)$, and by the inductive assumption we are done.

If $j_{1}+\varepsilon_{1}<i_{1}+\varepsilon_{2}$ then $j_{1}+\varepsilon_{1}$ is the smallest index in $\delta_{\varepsilon_{1}}(n) \delta_{\varepsilon_{2}}(p)$ and therefore, using $\mathcal{R}$, the word $\delta_{\varepsilon_{1}}(n) \delta_{\varepsilon_{2}}(p)$ can be rewritten the following way:

$$
\begin{gathered}
\delta_{\varepsilon_{1}}(n) \delta_{\varepsilon_{2}}(p)=x_{j_{t}+\varepsilon_{1}}^{-1} \ldots x_{j_{2}+\varepsilon_{1}}^{-1} x_{j_{1}+\varepsilon_{1}}^{-1} x_{i_{1}+\varepsilon_{1}} \ldots x_{i_{s}+\varepsilon_{2}} \stackrel{\mathcal{R}}{\longrightarrow} \\
\stackrel{\mathcal{R}}{\longrightarrow} x_{j_{t}+\varepsilon_{1}}^{-1} \ldots x_{j_{2}+\varepsilon_{1}}^{-1} x_{i_{1}+\varepsilon_{1}+1} \ldots x_{i_{s}+\varepsilon_{2}+1} x_{j_{1}+\varepsilon_{1}}^{-1}
\end{gathered}
$$

Note that since $j_{1}+\varepsilon_{1}$ is the smallest index in $\delta_{\varepsilon_{1}}(n) \delta_{\varepsilon_{2}}(p)$, the smallest index in $w=$ Merge $_{-,+}\left(x_{j_{t}}^{-1} \ldots x_{j_{2}}^{-1}, x_{i_{2}} \ldots x_{i_{s}}, \varepsilon_{1}, \varepsilon_{2}\right)$ is not less than $j_{1}+\varepsilon_{1}$. By the inductive assumption, $w$ is a seminormal form for $\delta_{\varepsilon_{1}}\left(x_{j_{t}}^{-1} \ldots x_{j_{2}}^{-1}\right) \delta_{\varepsilon_{2}}\left(x_{i_{2}} \ldots x_{i_{s}}\right)$. Therefore, $w x_{j_{1}+\varepsilon_{2}+1}^{-1}={ }_{F} \delta_{\varepsilon_{1}}(n) \delta_{\varepsilon_{2}}(p)$ and it is a seminormal form.

The last case where $j_{1}+\varepsilon_{1}>i_{1}+\varepsilon_{2}$ is treated similarly.

Using ideas from Algorithm 2 one can easily implement an algorithm merging positive words and an algorithm merging negative words, so that statements similar to Lemma 2 would hold. We will denote these two algorithms by Merge,$--\left(n_{1}, n_{2}, \varepsilon_{1}, \varepsilon_{2}\right)$ and $\operatorname{Merge}_{+,+}\left(p_{1}, p_{2}, \varepsilon_{1}, \varepsilon_{2}\right)$, respectively. Thus, computation of a seminormal form of a product of two arbitrary seminormal forms has the following form. 
Algorithm 3 (Seminormal form of a product of seminormal forms)

Signature. $w=\operatorname{Merge}\left(w_{1}, w_{2}\right)$.

INPUT. Seminormal forms $w_{1}$ and $w_{2}$.

Output. Seminormal form $w$ such that $w={ }_{F} w_{1} w_{2}$.

Computations.

A) Represent $w_{i}$ as a product of a positive and negative word $\left(w_{1}=p_{1} n_{1}\right.$ and $\left.w_{2}=p_{2} n_{2}\right)$.

B) Compute $w^{\prime}=$ Merge $_{-,+}\left(n_{1}, p_{2}, 0,0\right)$ and represent it as a product of a positive and negative word $w^{\prime}=p_{2}^{\prime} n_{1}^{\prime}$.

C) Compute $w^{\prime \prime}=$ Merge $_{+,+}\left(p_{1}, p_{2}^{\prime}, 0,0\right)$.

D) Compute $w^{\prime \prime \prime}=$ Merge $_{-,-}\left(n_{1}^{\prime}, n_{2}, 0,0\right)$.

E) Output $w^{\prime \prime} w^{\prime \prime \prime}$.

Lemma 3. For any pair of seminormal forms $w_{1}$ and $w_{2}$ the word $w=$ Merge $\left(w_{1}, w_{2}\right)$ is a seminormal form of the product $w_{1} w_{2}$. Moreover, the time-complexity of computing $w$ is bounded by $C\left(\left|w_{1}\right|+\left|w_{2}\right|\right)$ for some constant $C$.

Proof. Follows from Lemma 2.

\subsection{Seminormal Form Computation}

Algorithm 4 (Seminormal form)

Signature. $u=\operatorname{SemiNormalForm}(w)$.

InPUT. A word $w$ in generators of $F$.

Output. A seminormal form $u$ such that $u=w$ in $F$.

Computations.

A) If $|w| \leq 1$ then output $w$.

B) Represent $w$ as a product $w_{1} w_{2}$ such that $\left|w_{1}\right|-\left|w_{2}\right| \leq 1$.

C) Recursively compute

$u_{1}=\operatorname{SemiNormalForm}\left(w_{1}\right)$ and

$u_{2}=\operatorname{SemiNormalForm}\left(w_{2}\right)$.

D) Let $u=\operatorname{Merge}\left(u_{1}, u_{2}\right)$.

E) Output u.

Lemma 4. Let $w$ be a word in generators of $F$. The output of Algorithm 4 on $w$ is a seminormal form for $w$. The number of operations required for Algorithm 4 to terminate is $O(C|w| \log |w|)$, where $C$ is a constant independent of $w$.

Proof. The first statement can be proved by induction on the length of $w$. The base of the induction is the case where $|w|=1$. In this case $w$ is already in a seminormal form, and the output is correct. The induction step was proved in Lemma 3.

To prove the second statement we denote by $T(n)$ the number of steps required for Algorithm 4 to terminate on an input of length $n$. Then clearly

$$
T(n)=2 T\left(\frac{n}{2}\right)+C \cdot n,
$$

where the last summand $C \cdot n$ is the complexity of merging two seminormal forms with the sum of lengths at most $|n|$. It is an easy exercise to show that in this case $T(n)=O(C \cdot n \log n)$. 


\subsection{Normal Form Computation}

The next lemma suggests how a pair of generators contradicting the property (NF2) can be removed and how all such pairs can be found.

Lemma 5. Let $w=x_{i_{1}} \ldots x_{i_{s}} x_{j_{t}}^{-1} \ldots x_{j_{1}}^{-1}$ be a seminormal form, $\left(x_{i_{a}}, x_{j_{b}}^{-1}\right)$ be the pair of generators in $w$ which contradicts (NF2), where $a$ and $b$ are maximal with this property. Let

$$
w^{\prime}=x_{i_{1}} \ldots x_{i_{a-1}} \delta_{-1}\left(x_{i_{a+1}} \ldots x_{i_{s}} x_{j_{t}}^{-1} \ldots x_{j_{b+1}}^{-1}\right) x_{j_{b-1}}^{-1} \ldots x_{j_{1}}^{-1} .
$$

Then $w^{\prime}$ is in a seminormal form and $w={ }_{F} w^{\prime}$. Moreover, if $\left(x_{i_{c}}, x_{j_{d}}^{-1}\right)$ is the pair of generators in $w^{\prime}$ which contradicts (NF2) (where $a$ and $b$ are maximal with this property), then $c<a$ and $d<b$.

Proof. It follows from the definition of (NF2) and seminormal forms that all indices in $x_{i_{a+1}} \ldots x_{i_{s}} x_{j_{t}}^{-1} \ldots x_{j_{b+1}}^{-1}$ are greater than $i_{a}+1$ and, therefore, indices in $\delta_{-1}\left(x_{i_{a+1}} \ldots x_{i_{s}} x_{j_{t}}^{-1} \ldots x_{j_{b+1}}^{-1}\right)$ are greater than $i_{a}$. Now it is clear that $w^{\prime}$ is a seminormal form. Then doing rewrites opposite to rewrites from $\mathcal{R}$ we can get the word $w^{\prime}$ from the word $w$. Thus, $w={ }_{F} w^{\prime}$.

There are two possible cases: either $c>a$ and $d>b$ or $c<a$ and $d<b$. We need to show that the former case is, in fact, impossible. Assume, by way of contradiction, that $c>a$ and $d>b$. Now observe that if $\left(x_{i_{a}}, x_{j_{b}}^{-1}\right)$ is a pair of generators in $w$ contradicting (NF2), then $\left(x_{i_{a}+\varepsilon}, x_{j_{b}+\varepsilon}^{-1}\right)$ contradicts (NF2) in $\delta_{\varepsilon}(w)$. Therefore, inequalities $c>a$ and $d>b$ contradict the choice of $a$ and $b$.

By Lemma 5 we can start looking for bad pairs in a seminormal form starting from the middle of a word. The next algorithm implements this idea. The algorithm is in two parts. The first part finds all "bad" pairs starting from the middle of a given $w$, and the second part applies $\delta_{\varepsilon}$ to segments where it is required. A notable feature of Algorithm 5 is that it does not apply the operator $\delta_{-1}$ immediately (as in $w^{\prime}$ of Lemma 5) when a bad pair is found, but instead, it keeps the information about how indices must be changed later. This information is accumulated in two sequences (stacks), one for the positive subword of $w$, the other one for the negative subword of $w$. Also, in Algorithm 5, the size of stack $S_{1}$ (or $S_{2}$ ) equals the length of an auxiliary word $w_{1}$ (resp. $w_{2}$ ). Therefore, at step B), $x_{a}$ (resp. $x_{b}$ ) is defined if and only if $\varepsilon_{1}$ (resp. $\left.\varepsilon_{2}\right)$ is defined.

Algorithm 5 (Erasing bad pairs from a seminormal form)

Signature. $w=$ EraseBadPairs $(u)$.

INPUT. A seminormal form $u=x_{i_{1}} \ldots x_{i_{s}} x_{j_{t}}^{-1} \ldots x_{j_{1}}^{-1}$.

Output. A word $w$ which is the normal form of $u$.

InitializATION. Let $\delta=0, \delta_{1}=0, \delta_{2}=0, w_{1}=1$, and $w_{2}=1$. Let $u_{1}=$ $x_{i_{1}} \ldots x_{i_{s}}$ and $u_{2}=x_{j_{t}}^{-1} \ldots x_{j_{1}}^{-1}$ be the positive and negative parts of $u$. Additionally, we set up two empty stacks $S_{1}$ and $S_{2}$. 


\section{Computations.}

A. Let the current $u_{1}=x_{i_{1}} \ldots x_{i_{s}}$ and $u_{2}=x_{j_{t}}^{-1} \ldots x_{j_{1}}^{-1}$.

B. Let $x_{a}$ be the leftmost letter of $w_{1}, x_{b}$ the rightmost letter of $w_{2}$, and $\varepsilon_{i}$ $(i=1,2)$ the top element of $S_{i}$, i.e., the last element that was put there. If any of these values does not exist (because, say, $S_{i}$ is empty), then the corresponding variable is not defined.

1) If $s>0$ and $\left(t=0\right.$ or $\left.i_{s}>j_{t}\right)$, then:

a) multiply $w_{1}$ on the left by $x_{i_{s}}$ (i.e. $\left.w_{1} \leftarrow x_{i_{s}} w_{1}\right)$;

b) erase $x_{i_{s}}$ from $u_{1}$;

c) push 0 into $S_{1}$;

d) goto 5).

2) If $t>0$ and $\left(s=0\right.$ or $\left.j_{t}>i_{s}\right)$, then:

a) multiply $w_{2}$ on the right by $x_{j_{t}}^{-1}$ (i.e. $w_{2} \leftarrow w_{2} x_{j_{t}}^{-1}$ );

b) erase $x_{j_{t}}^{-1}$ from $u_{2}$;

c) push 0 into $S_{2}$;

d) goto 5).

3) If $i_{s}=j_{t}$ and (the numbers $a-\varepsilon_{1}$ and $b-\varepsilon_{2}$ (those that are defined) are not equal to $i_{s}$ or $\left.i_{s}+1\right)$, then:

a) erase $x_{i_{s}}$ from $u_{1}$;

b) erase $x_{j_{t}}^{-1}$ from $u_{2}$;

c) if $S_{1}$ is not empty, increase the top element of $S_{1}$;

d) if $S_{2}$ is not empty, increase the top element of $S_{2}$;

e) goto 5).

4) If 1)-3) are not applicable (when $i_{s}=j_{t}$ and (one of the numbers $a-\varepsilon_{1}$, $b-\varepsilon_{2}$ is defined and is equal to either $i_{s}$ or $\left.i_{s}+1\right)$ ), then:

a) multiply $w_{1}$ on the left by $x_{i_{s}}$ (i.e. $\left.w_{1} \leftarrow x_{i_{s}} w_{1}\right)$;

b) multiply $w_{2}$ on the right by $x_{j_{t}}^{-1}$ (i.e. $w_{2} \leftarrow w_{2} x_{j_{t}}^{-1}$ );

c) erase $x_{i_{s}}$ from $u_{1}$;

d) erase $x_{j_{t}}^{-1}$ from $u_{2}$;

e) push 0 into $S_{1}$;

f) push 0 into $S_{2}$;

g) goto 5).

5) If $u_{1}$ or $u_{2}$ is not empty then goto 1 ).

C. While $w_{1}$ is not empty:

1) let $x_{i_{1}}$ be the first letter of $w_{1}$ (i.e. $\left.w_{1}=x_{i_{1}} \cdot w_{1}^{\prime}\right)$;

2) take (pop) c from the top of $S_{1}$ and add to $\delta_{1}$ (i.e. $\delta_{1} \leftarrow \delta_{1}+c$ );

3) multiply $u_{1}$ on the right by $x_{i_{1}-\delta_{1}}$ (i.e. $u_{1} \leftarrow u_{1} x_{i_{1}-\delta_{1}}$ );

4) erase $x_{i_{1}}$ from $w_{1}$.

D. While $w_{2}$ is not empty:

1) let $x_{j_{1}}^{-1}$ be the last letter of $w_{2}$ (i.e. $\left.w_{2}=w_{2}^{\prime} \cdot x_{j_{1}}^{-1}\right)$;

2) take (pop) c from the top of $S_{2}$ and add to $\delta_{2}$ (i.e. $\delta_{2} \leftarrow \delta_{2}+c$ );

3) multiply $u_{2}$ on the left by $x_{j_{1}-\delta_{2}}^{-1}$ (i.e. $\left.u_{2} \leftarrow x_{j_{1}-\delta_{2}}^{-1} u_{2}\right)$;

4) erase $x_{j_{1}}^{-1}$ from $w_{2}$.

E. Return $u_{1} u_{2}$. 
Proposition 3. The output of Algorithm 5 is the normal form $w$ of a seminormal form $u$. The number of operations required for Algorithm 5 to terminate is bounded by $D \cdot|u|$, where $D$ is a constant independent of $u$.

Proof. The first statement follows from Lemma 5. The time estimate is obvious from the algorithm since the words $u_{1}, u_{2}$ are processed letter-by-letter, and no letter is processed more than once.

As a corollary, we get the main result of this section:

Theorem 1. In Thompson's group F, the normal form of a given word $w$ can be computed in time $O(|w| \log |w|)$.

\section{References}

1. I. Anshel, M. Anshel, D. Goldfeld, An algebraic method for public-key cryptography, Math. Res. Lett. 6 (1999), 287-291.

2. J. W. Cannon, W. J. Floyd, and W. R. Parry, Introductory notes on Richard Thompson's groups, L'Enseignement Mathematique (2) 42 (1996), 215-256.

3. J. C. Cha, K. H. Ko, S. J. Lee, J. W. Han, J. H. Cheon, An Efficient Implementation of Braid Groups, ASIACRYPT 2001, Lecture Notes in Comput. Sci. 2248 (2001), $144-156$.

4. D. Hofheinz and R. Steinwandt, A practical attack on some braid group based cryptographic primitives, in Public Key Cryptography, 6th International Workshop on Practice and Theory in Public Key Cryptography, PKC 2003 Proceedings, Y.G. Desmedt, ed., Lecture Notes in Computer Science 2567, pp. 187-198, Springer, 2002.

5. J. Hughes and A. Tannenbaum, Length-based attacks for certain group based encryption rewriting systems, Workshop SECI02 Securitè de la Communication sur Intenet, September 2002, Tunis, Tunisia.

http://www.storagetek.com/hughes/

6. K. H. Ko, S. J. Lee, J. H. Cheon, J. W. Han, J. Kang, C. Park, New public-key cryptosystem using braid groups, Advances in cryptology - CRYPTO 2000 (Santa Barbara, CA), 166-183, Lecture Notes in Comput. Sci. 1880, Springer, Berlin, 2000.

7. V. Shpilrain, Assessing security of some group based cryptosystems, Contemp. Math., Amer. Math. Soc. 360 (2004), 167-177.

8. V. Shpilrain and A. Ushakov, The conjugacy search problem in public key cryptography: unnecessary and insufficient, Applicable Algebra in Engineering, Communication and Computing, to appear.

http://eprint.iacr.org/2004/321/

9. V. Shpilrain and G. Zapata, Combinatorial group theory and public key cryptography, Applicable Algebra in Engineering, Communication and Computing, to appear.

10. C. Sims, Computation with finitely presented groups, Encyclopedia of Mathematics and its Applications, 48. Cambridge University Press, Cambridge, 1994. 